\title{
VICTIMES OU ACTIONS: ITINÉRAIRES OCCULTES D'ETA
}

\section{The Transitional Devise: From the Administration of Uncertainty to the Emergence of New Socialities}

\author{
Caroline Guibet Lafaye* \\ Centre National de la Recherche Scientifique-Centre Émile Durkheim (UMR 5116) (Francia)
}

\begin{abstract}
Mots-clefs
ETA

Violence

clandestine

Stratégie militaire

Pays Basque

Base de données sentons une base de données originale de ses actions à partir de laquelle il s'avère possible de mettre en évidence les limites de certains choix méthodologiques et de proposer une analyse affinée des options stratégiques du groupe sur ses soixante années d'existence. La présente étude contribue ainsi à mettre au jour des aspects occultés par la littérature de la stratégie du collectif, qu'il soit question des modalités de sa réponse à l'antiterrorisme, du soutien aux mouvements sociaux des années 1980 aux années 2000, ou des attaques contre les intérêts économiques espagnols, dommageables sur le plan matériel mais peu coûteuses en vies humaines.
\end{abstract}

RÉSUMÉ: Euskadi Ta Askatasuna (ETA) est l'organisation clandestine qui a eu, en Europe occidentale, la plus longue durée de vie. À ce jour, toutes les études statistiques la concernant ont été produites à partir du recensement de ses victimes. Dans cet article, nous pré-

RESUMEN: Euskadi Ta Askatasuna (ETA) es la organización clandestina que ha tenido la mayor duración de vida en Europa Occidental. Hasta la fecha, todos los estudios estadísticos relativos a ella se han realizado sobre la base del censo de sus víctimas. En este artículo presentamos una base de datos original construida en torno a sus acciones que permite poner de relieve los límites de ciertas opciones metodológicas y proponer un análisis refi-

Palabras clave

ETA nado de las opciones estratégicas del grupo a lo largo de sus sesenta años de existencia. EI

Violencia presente estudio contribuye así a descubrir aspectos de la estrategia del colectivo que se clandestina Estrategia militar País Vasco Base de datos han pasado por alto en la literatura ya sea las modalidades de su respuesta a la lucha contra el terrorismo, el apoyo a los movimientos sociales desde los años ochenta a los años 2000, o los ataques contra los intereses económicos españoles, que son perjudiciales en términos materiales pero no costosos en términos de vidas humanas.

\footnotetext{
* Correspondence à / Correspondence to: Caroline Guibet Lafaye. Centre National de la Recherche Scientifique-Centre Émile (UMR 5116). 3 ter Place de la Victoire (33076-Bordeaux, Francia) - caroline.guibetlafaye@u-bordeaux.fr - http://orcid.org/0000-0002-2114-7605.

Comment citer / How to cite: Guibet Lafaye, Caroline (2021). "Victimes ou actions : itinéraires occultes d'ETA ». Papeles del CEIC, vol. 2021/1, papier 245, 1-22. (http://dx.doi.org/10.1387/pceic.22161).
}

Date de réception: octobre, 2020 / Date d'acceptation: décembre, 2020.

ISSN 1695-6494 / (C) 2021 UPV/EHU 
Le terrorisme, lorsqu'il s'abat sur un pays, alimente nombre d'articles de presse. Néanmoins les chiffres à partir desquels on aborde ce phénomène s'avèrent souvent sujets à contestation. Nous en ferons la démonstration pour le cas du groupe clandestin Euskadi Ta Askatasuna (ETA). ETA est créée fin 1958 par des étudiants basques réunis autour du journal Ekin et proches du Parti Nationaliste Basque (PNV). L'existence du groupe est rendue publique le 31 juillet 1959'. De son origine à son autodissolution le 11 mai 2018, ETA s'est proclamée " organisation socialiste révolutionnaire basque de libération nationale $॥$.

Selon les sources d'information consultées, le nombre de victimes mortelles causées par cette dernière varie au même titre que l'authenticité de la responsabilité des actions commises $^{2}$. Le ministère de l'Intérieur espagnol recense 1.380 décès dus au terrorisme entre 1960 et 2014, dont 829 seraient le fait d'ETA, 82 des Groupes de Résistance Antifasciste $1^{\text {er }}$ Octobre (GRAPO), 232 de l'islam politique violent et du terrorisme international et 239 de groupes dits " autres ". 842 victimes ont fait l'objet d'une indemnisation publique en vertu de la loi 29/2011 du 22 septembre sur la reconnaissance et la protection intégrale des victimes du terrorisme ou de la loi de solidarité de 1999. Un rapport de la Direction générale au soutien des victimes du terrorisme du ministère de l'Intérieur, publié en 2010, recense 856 victimes mortelles d'ETA. La Fondation pour les victimes du terrorisme (FVT) propose le chiffre de 829 (et 85 pour les GRAPO) alors que le Collectif des Victimes du Terrorisme (COVITE) estime à 858 le nombre de personnes tuées par ETA. Dans un conflit politique, il est particulièrement délicat d'étudier l'un des belligérants à partir des chiffres produits par son " ennemi " politique. Or, dans le cas d'un groupe clandestin en activité l'accès aux sources est particulièrement délicat. Selon les sources dont on s'inspire et les analyses qu'elles nourrissent, les phénomènes de "cadrage " et d'interprétation des acteurs politiques ainsi que de leurs stratégies varient (Goffman, 1974 ; Benford et Snow, 2012).

De sa constitution à son autodissolution, ETA s'est perpétuée dans un contexte où la violence politique légale et illégale était de mise des deux côtés des Pyrénées. Entre 1960 et 2014, on comptabilise, au Pays basque, 927 victimes mortelles dont 67 sont le fait de l'antiterrorisme (Fonseca, 2014: 55). De 1960 à 2013, entre 2.300 et 2.600 personnes sont blessées par les différentes branches clandestines se revendiquant d'ETA (ETA-pm, ETA-m, ETA-VII, ETA-VIII assemblée) ainsi que de l'extrême gauche. 746 doivent être attribuées aux forces de sécurité de l'État et 426 aux groupes paramilitaires et d'extrême droite (Carmena et al., 2013 : 14). En dépit du nombre de victimes réalisées, ETA est considérée comme une organisation ayant pratiqué la violence politique de façon " relativement sélective ". La corrélation par trimestre entre le nombre de décès et le nombre d'attaques mortelles est de 0,93 (Sánchez-Cuenca, 2009 : 7). Des études antérieures ont montré que fin 2007, ETA avait conduit 597 attaques mortelles et tué 836 personnes avec une moyenne d'1,4 décès par attaque mortelle (ibidem).

1 Voir : Madariaga, Punto y Hora in Euskal Herria, 13 juillet 1984, 357: 8. Jusqu'au début de l'année 1959, le groupe ne diffuse ni son nom ni ne fait connaître son existence par mesure de sécurité.

2 Dans ce qui suit, nous utiliserons les termes d' " actions ", d'" opérations ", d' " attaques " plutôt que d' " attentats " dans un souci de neutralité axiologique et pour nous placer dans une perspective simplement descriptive. Nous renvoyons sur ce point à la littérature sur le terrorisme qui souligne l'écueil de l'usage d'un vocabulaire dont la finalité est normative et politique plutôt qu'il ne sert un objectif de description et d'explication d'un phénomène social (Tilly, 2004: 5). 
Comme nous y reviendrons de façon détaillée, l'objectivation statistique tend à produire une image de la réalité sociale (Desrosières, 2008). Jusqu'à présent, les seules statistiques officielles visant à rendre compte de l'activité armée d'ETA ont été publiées par des agences gouvernementales. II s'avère pourtant possible de saisir cette activité à partir de sources officielles quoique non exclusivement gouvernementales. Dès lors, une toute autre représentation de la stratégie clandestine d'ETA s'esquisse. Afin de souligner l'écart d'interprétation qu'autorise la référence à des données statistiques, nous comparerons les bases de données exploitées jusqu'à présent par les travaux quantitatifs consacrés à ETA ${ }^{3}$ et la base que nous avons réalisée. Cette double référence conduit à des divergences d'interprétation majeures quant à l'activité stratégique d'un groupe armé illégal auquel l'accès est structurellement difficile. La stratégie de ce type de groupes se laisse appréhender à la fois par les déclarations qu'ils rendent publiques aussi bien que par les opérations menées. Toutefois l'élaboration de données susceptibles de témoigner de ces dernières résulte de choix méthodologiques qui ne sont pas neutres, selon que l'attention est portée aux actions planifiées (menées à bien ou déjouées), aux victimes (morts et/ou blessés) voire aux membres impliqués dans ces opérations ou dans les arrestations qu'elles ont pu susciter. En particulier s'agissant, d'une part, de l'évolution des options stratégiques d'ETA à travers le temps et, d'autre part, de l'effet des politiques antiterroristes sur son activité, les résultats que proposent l'une et l'autre bases conduisent à des conclusions divergentes. Le cas d'ETA et de son activité armée constitue une illustration de la façon dont les statistiques peuvent être utilisées dans le débat public et dans la production de représentations par des acteurs impliqués dans un conflit politique durable.

Après avoir rappelé brièvement l'histoire du groupe, nous exposerons les éléments sur lesquels nous nous sommes appuyés pour constituer une base de données originale des opérations d'ETA et pas non exclusivement de ses victimes. Cette base compte 3.156 items ${ }^{4}$. Elle nous permettra de dévoiler des aspects de la stratégie du collectif négligés par la littérature, qu'il soit question de l'accroissement des opérations militaires sans victime en représailles à l'antiterrorisme, du soutien aux mouvements sociaux des années 1980 aux années 2000 ou de la récurrence de l'attaque contre les intérêts économiques espagnols, dommageable sur le plan matériel mais peu coûteuse en vies humaines.

3 De La Calle et Sanchez-Cuenca (2004) exploitent la base Domestic Terrorist Victims, extraite d'une base de données plus large qui couvre 18 pays d'Europe occidentale sur la période 1965-2000. Elle s'appuie sur des sources en six langues différentes (monographies sur des organisations terroristes particulières, journaux nationaux, documents rédigés par les groupes clandestins, données recueillies par les États, pages internet contenant des informations sur les victimes du terrorisme). Pour une lecture critique de l'élaboration des bases de données relative à ETA, voir : Guibet Lafaye et Brochard (2020, 2021).

4 Les " opérations " d'ETA retenues ne sont pas seulement celles qui ont été revendiquées car toutes ne l'ont pas été. Certaines, du fait des cibles visées, ne laissaient pas de doute quant à leur origine. Le groupe a assumé, en avril 2018, la responsabilité de 2.606 actions (Mediabask, 6/11/2018). Le différentiel tient au fait que nous nous sommes également appuyés sur des sources journalistiques. 


\subsection{Bref aperçu de l'histoire d'ETA}

Sans entrer dans les détails d'une histoire de plus de cinq décennies, nous rappellerons brièvement les phases de l'évolution du groupe clandestin ${ }^{5}$. ETA s'est constituée en organisation clandestine en décembre 1958 et a fait connaître son existence le 31 juillet 1959. Elle s'est formée à l'initiative d'étudiants basques œuvrant dans le magazine Ekin. Ce dernier visait à promouvoir l'identité basque et son étude. ETA a connu plusieurs phases, la première coïncidant avec une période de résistance contre le régime franquiste (1959-1975). L'action du groupe a connu sa plus forte intensité durant la transition de l'Espagne vers un régime démocratique (1977-1979) puis au cours des premières années du régime socialiste investi le $1^{\mathrm{er}}$ décembre 1982 (1982-1986). La fin des années 1980 et les années 1990 sont marquées par plusieurs processus de négociation entre les émissaires du groupe et ceux du gouvernement, sans réel succès, ainsi que par plusieurs trêves ou cessez-le-feu unilatéraux. La littérature a souvent retenu l'année 1992 comme épisode clef du fait de l'arrestation de l'exécutif d'ETA le 29 mars 1992 à Bidart (Pyrénées-Atlantiques) (Sánchez-Cuenca, 2009 : 22). Bien qu'au cours des années 2000 le collectif ait pris l'option de réduire le nombre de victimes, en revanche l'intensité de ses interventions était en 2000-2001 comparable à ce qu'elle était en 1987-1988 (Guibet Lafaye et Brochard, 2021). ETA continue de mener, de façon sporadique, des opérations jusqu'en 2009-2010 tout en cherchant une solution négociée au conflit au Pays basque avec le gouvernement espagnol. L'évolution du contexte international et de la situation dans ce territoire, les transformations internes au groupe ainsi que de la gauche abertzale (patriote) ont conduit ETA à prononcer son autodissolution le 3 mai 2018.

\subsection{Constitution de la base de données et description des sources utilisées}

La base de données que nous avons réalisée ${ }^{6}$, recensant l'ensemble des actions menées par ETA, s'appuie sur plusieurs types de sources :

1. Elle s'est en premier lieu nourrie de bases de données existantes notamment de celle du Global Terrorism Database (GTD) ${ }^{7}$ mais pas exclusivement (voir Annexe 1).

2. Nous avons également retenu les bases de données répertoriant les victimes du terrorisme basque en prenant soin de ne retenir que celles faites par ETA (ETA-m et ETA-pm) à l'exclusion de tout autre groupe ${ }^{8}$.

5 Pour une histoire extensive d'ETA, voir : Nuñez Astrain (1993) ainsi que Massey (2010) et Giacopucci (1997).

En collaboration avec Pierre Brochard (LAMOP-Université Paris I Panthéon-Sorbonne).

Nous avons procédé à un travail à la fois de nettoyage et de complément de la base GTD dans la mesure où, d'une part, aucune donnée n'a été collectée pour l'année 1993 et, d'autre part comme nous l'avons souligné, elle compile au moins 79 actions qui ne sont pas le fait d'ETA (qu'elles soient imputables à Iparretarrak, Iraultza, Gatazka, à la kale borroka (i.e. à la guérilla urbaine des années 1990-2000) voire associe aux actions d'ETA celles des groupes paramilitaires d'extrême droite qui conduisent à la mort de membres d'ETA (ex. attentats du 29 mai 1994 à Muskiz et Artxanda). Dans cette base, la responsabilité de 321 actions n'a pu être éclaircie et ne peut donc être imputée à ETA. La base que nous avons constituée est originale et disponible sur demande.

8 Il s'agit du Colectivo de Victimas del Terrorismo [COVITE, https://covite.org], de l'Asociación de Víctimas del Terrorismo [AVT, https://avt.org/es], d'AROVITE [Archivo Onligne sobre la Violencia Terrorista en Euskadi : https:// www.arovite.com/es/bases-de-datos/], des documents produits par le gouvernement basque : "Clarificación 
3. Nous avons exploité les quotidiens nationaux et régionaux, en castillan, français, anglais et en langue basque (euskara), et plus généralement la presse (en réalisant des extractions à partir de la mention " ETA » et en excluant tous les items non pertinents), opération omise par GTD9.

4. Les sources gouvernementales du ministère de l'Intérieur espagnol.

5. Les documents produits par la gauche abertzale et par l'organisation clandestine elle-même.

Les sources ont été systématiquement croisées pour pallier l'absence de revendication et l'attribution fautive au groupe clandestin d'actions non revendiquées. Elles sont systématiquement répertoriées en Annexe 1. Nous avons retenu à la fois les actions militaires, qu'elles aient réussi ou échoué (cas des bombes désamorcées ou des engins qui n'ont pas explosé), mais également, pour les premières décennies d'existence d'ETA, toutes les actions illégales susceptibles d'attirer la répression. Les premières opérations clandestines n'ont pas fait de victime et peuvent sembler insignifiantes qu'il s'agisse de graffiti, d'accrochages du drapeau basque (ikurriña) alors interdit, de distributions de tracts. Pourtant les fondateurs du groupe ${ }^{10}$ ainsi que les documents officiels rappellent les risques qu'encourraient leurs auteurs ${ }^{11}$. Ces actions ont été fondatrices du groupe clandestin. Elles ont éveillé la conscience de la population sur la possibilité d'une résistance à la répression. Du fait de leur illégalité, elles ont déchaîné des vagues d'arrestations ${ }^{12}$ qui justifient qu'elles figurent dans la base de données.

Dans l'exploitation de cette base de données et contrairement à ce qui a souvent été le cas antérieurement (Barros, 2003 ; De la Calle et Sanchez-Cuenca, 2004, 2006 ; Barros, Passos, et Gil-Alana, 2006), nous avons fait le choix d'une périodisation par semestres plutôt qu'annuelle. Elle permet une approche plus fine des évolutions stratégiques du groupe ${ }^{13}$. En particulier, il s'avère possible de lire les effets d'événements politiques singuliers (élections, négociations, arrestations, etc.) sur les options politico-stratégiques de l'organisation.

La mobilisation de statistiques dans le monde contemporain véhicule des enjeux politiques majeurs. La quantification, tenue pour une marque d'objectivité, de rigueur et d'impartialité se voit aujourd'hui fréquemment mobilisée dans le débat politique, notamment sous la forme de mesure de l'opinion publique. "L'argument statistique " (Desrosières, 2008) constitue ainsi un outil de gouvernement et le point d'appui de nouvelles formes de "gouvernementalité " (Foucault, 2004). Pourtant le passage par la quantification, loin de fournir une image neutre et objective des phénomènes, les transforme. La quantification ne propose pas un reflet du monde mais crée une nouvelle façon de le penser, de le représenter, de l'exprimer

del pasado ", 2016. Ces associations de victimes, à l'origine constituées de familles ayant subi la violence politique d'ETA, rassemblent aujourd'hui les victimes et familles de toute forme de violence politique quelle que soit son origine. Leur influence sur la scène publique et politique est particulièrement remarquable (Truc, 2012).

9 En particulier les quotidiens : El País, El Mundo, Diario Vasco, El Correo, ABC, El Norte de Castilla, EITB, EI Periódico, La Información.

10 Voir : Madariaga, "Así nació ETA », Punto y Hora, 25 Aniversario, 13 juillet 1984: 7-9 ; Zunbeltz, 1968 : 106-107, cité in Nuñez, 1993, I: 92-93.

11 "La quema de la bandera en un edificio oficial, a plena luz del día, en un San Sebastián donde el veraneo reunía a lo más selecto del Régimen y del aparato fascista, suponía una operación madurada, calculada y con cierto riesgo. " (Jon Nicolás, Documentos Y, 1979, cité in Nuñez, 1993, I: 60 ; voir aussi : Zunbeltz, 1968 : 106-107, cité in Nuñez, 1993, I: 92-93).

12 Citons pour seul exemple l'adoption du décret 1794/1960 sur la « Rébellion Militaire, le banditisme et le terrorisme " (Rebelion Militar, Bandidaje y Terrorismo) le 21 septembre 1960.

13 Seul Sanchez-Cuenca (2009) a proposé une analyse trimestrielle des victimes mortelles faites par ETA. 
et d'agir sur lui (Desrosières, 2008) que ce soit par ses résultats ou par leurs usages argumentatifs. Ainsi, en raison des effets cognitifs et sociaux des dispositifs de quantification, celle-ci peut se targuer d'une emprise dans l'espace public. La statistique, qui fournit une représentation simplifiée et supposée objective des faits sociaux, constitue un outil efficace pour faire émerger certains phénomènes comme des problèmes publics et orienter leur traitement ${ }^{14}$. Les statistiques produisent de la normativité et contribuent à nourrir des notions d'objectivité et de juste représentation (Samson, 2014). Elles participent de la construction des représentations dans l'opinion publique et sont bien souvent à l'origine de l'action politique et/ou des politiques publiques. De nos jours, le gouvernement par les nombres est devenu une norme dans bien des pays. Non seulement les statistiques sont un mode de communication entre différents acteurs (Davidshofer, Tawfik et Hagmann, 2016 ; Speich Chassé, 2017) ${ }^{15}$ mais la communication statistique s'avère actuellement être une importante source de pouvoir politique. On le vérifie également concernant la question du terrorisme ${ }^{16}$. Pourtant cet outil présente des limites. Dans le champ du terrorisme en particulier, on a montré que certaines bases de données existantes, conçues pour l'appréhender, parvenaient mal à en rendre compte, y compris d'un point de vue quantitatif (McCaffery, Richardson et Bélanger , 2016). Non seulement la détermination de "groupes terroristes " constitue une catégorie problématique dans une base comme celle de GTD (Miller, 2016) ${ }^{17}$ mais le phénomène terroriste suppose de tenir compte à la fois du contexte dans lequel il opère ainsi que des motivations et de l'idéologie des infracteurs, ce que les statistiques canadiennes et les 13 codes d'infraction du Uniform Crime Reporting (UCR), par exemple, échouent à appréhender (McCaffery, Richardson et Bélanger, 2016). En outre, un attentat déjoué peut avoir des effets comparables à celui d'un attentat réussi en termes d'incidence sur la population ou le groupe social visé.

Si les bases de données des victimes d'ETA permettent de cerner l'évolution de ses cibles, dans une logique d' " attaque au cœur de l'État » ou, à tout le moins, de ses représentants à partir du début des années 1990, en revanche elles occultent les fronts d'action principaux de l'organisation. L'identification des actions du groupe contribue en revanche à les mettre en lumière (voir Tableau 1, Tableau 6). Ces fronts relèvent des champs politiques, sociaux et économiques. L'élucidation des opérations réalisées et de leurs raisons contribue à porter une lumière sur l'organisation clandestine qui n'est pas exclusivement tributaire de "l'approche par les victimes ", dominante dans la littérature scientifique espagnole. Nous envisagerons successive-

14 Sur le cas de l'éducation nationale et de la santé publique en France, voir : Foucart, 2001. Concernant les politiques sécuritaires déployées en Suisse, voir : Davidshofer, Tawfik et Hagmann, 2016.

15 On a même parlé, dans certains cas, de " guerre de chiffres " (François et Machiels, 2007) lorsqu'ont pu être mises en évidence une construction et une évolution de séries statistiques, en fonction des préoccupations des classes dirigeantes belges successives, ainsi que les efforts des acteurs pour présenter ces données à leur avantage, allant du simple discours orienté à la manipulation pure et simple.

16 Voir : Bacevich (2001) sur le rapport annuel du département d'État américain Patterns in Global Terrorism (https://www.csis.org/analysis/patterns-global-terrorism-1970-2016).

17 Il n'est pas certain qu'il soit pertinent d'assimiler sous cette même catégorie des organisations terroristes, des organisations criminelles, des groupes d'insurgés, des partis politiques qui se livrent occasionnellement à des actes terroristes, des partis politiques qui recourent régulièrement à ce type d'actions, des organisations terroristes qui se sont transformées en partis politiques, des groupes dissidents, des "labels " à usage unique d'auteurs d'attentats, des individus non affiliés ou faiblement affiliés à un groupe mais qui s'en revendiquent. 
ment la « riposte » contre l'État français, la lutte contre les Grands Projets Inutiles et l'attaque contre les intérêts économiques de l'État espagnol sous la forme des « campagnes d'été ».

Tableau 1

Répartition des actions d'ETA menées des origines à sa dissolution

\begin{tabular}{|c|c|c|}
\hline Catégories ${ }^{18}$ & Victimes & Actions menées \\
\hline Actions symboliques & 21 & 102 \\
\hline Banque & 9 & 212 \\
\hline Bâtiments publics & 0 & 198 \\
\hline Civils & 4 & 9 \\
\hline Drogue & 49 & 96 \\
\hline Entreprises (lutte sociale) & 1 & 29 \\
\hline Entreprises privées & 1 & 29 \\
\hline Forces de sécurité espagnoles (FSE) & 579 & 787 \\
\hline Impôt révolutionnaire & 16 & 163 \\
\hline Informateurs de la police & 34 & 48 \\
\hline Infrastructure & 12 & 156 \\
\hline Infrastructure (lutte sociale) & 10 & 400 \\
\hline Intérêts américains & 0 & 6 \\
\hline Intérêts français & 4 & 360 \\
\hline Journalistes $\&$ Media & 5 & 38 \\
\hline Lieux publics & 1 & 37 \\
\hline Personnel politique & 42 & 71 \\
\hline Prison & 7 & 18 \\
\hline Propriétés privées & 1 & 2 \\
\hline Réponse à l'antiterrorisme & 20 & 58 \\
\hline Représentants de l'État & 22 & 55 \\
\hline Réquisitions & 0 & 53 \\
\hline Tourisme & 1 & 220 \\
\hline Non identifiables & 0 & 9 \\
\hline Total & 839 & 3.156 \\
\hline
\end{tabular}

18 Afin d'ordonner la pluralité des opérations menées par ETA, nous avons procédé à leur regroupement en plusieurs catégories. Certaines appellent quelques éclaircissements. Sous la catégorie " Entreprises (lutte sociale) ", nous plaçons les actions menées en soutien à des mouvements sociaux, portant des revendications de type syndical notamment. "Infrastructure » désigne des cibles telles que les aéroports, les structures ferroviaires, les autoroutes ou les ponts, les télécommunications, les raffineries de pétrole, et qui sont attaquées en vue de me mettre à mal l'État espagnol. S'en distinguent, en revanche, les opérations visant les « Infrastructures (lutte sociale) ", c'est-à-dire le projet de centrale nucléaire de Lemoiz, l'autoroute de Leizarán et les entreprises impliquées dans ces chantiers, au premier chef desquels Iberduero. 


\subsection{Effets contre-productifs de l'antiterroriste illégal}

Les actions contre les intérêts français, qui sont rarement mortelles ${ }^{19}$, se concentrent spécifiquement sur la période 1984-1991 et permettent d'apprécier l'effet des politiques antiterroriste franco-espagnole. Elles sont au nombre de 360.

Tableau 2

Opérations contre des intérêts français au cours de l'histoire d'ETA

\begin{tabular}{|c|c|}
\hline Périodes & Actions menées \\
\hline $1959-1978$ & 0 \\
\hline $1979-1983$ & 40 \\
\hline $1984-1987$ & 178 \\
\hline $1988-1991$ & 131 \\
\hline $1992-2001$ & 11 \\
\hline $2002-2018$ & 0 \\
\hline
\end{tabular}

L'analyse des actions perpétrées par ETA autorise une appréciation des effets des politiques antiterroristes illégales et de coopération internationale de l'État espagnol à partir des années 1980 à 2000, notamment en termes de dissuasion (Barros, 2003). Elle soulève les effets contre-productifs de l'antiterroriste illégal sur les actions d'ETA puisqu'elle tend à les accroître sous la modalité des représailles à la "guerre sale " menée sur le territoire français (Guittet, 2000 ; Massey, 2010: 149-195). Cette dernière fait référence aux organisations clandestines secrètes, telles la Triple A (Alliance Apostolique Anticommuniste), I'ATE (Antiterrorisme ETA), I'ANE (Action Nationale Espagnole), les GAE (Groupes Antiterroristes Espagnols), les Guérilleros du Christ-Roi et surtout les GAL (Groupes Antiterroristes de Libération) qui ont œuvré, depuis le régime de Franco, contre l'activisme basque. Dès les années 1960-1970, la répression n'incombe pas seulement aux appareils de sécurité de l'État espagnol mais se voit également assumée par ces groupes (Guittet, 2000 : 24). Les revendications indépendantistes sont perçues comme contraires à l'intérêt de la nation. Cette conviction, associée à la thèse selon laquelle "todo vale para acabar con ETA " (tout est bon pour en finir avec ETA) et au fait que jusque dans les années quatre-vingt, les structures policières et militaires de l'État espagnol restent tributaires d'un appareil militaire conçu et composé par le régime franquiste et pour le régime de Franco (Lopez Garrido, 1987), expliquent l'émergence des GAL. Or à l'exception du double assassinat de Lasa et Zabala le 16 octobre 1983, de celui de Santi Brouard le 20 novembre 1984 et de la séquestration de Segundo Marey le 14 décembre 1983, les trentecinq opérations connues des GAL ont toutes eu lieu sur le sol français, entre Bayonne, Hendaye et Saint-Martin d'Arrossa. Non seulement les GAL sont une émanation des structures politiques et sécuritaires espagnoles mais ils interviennent également comme un instrument de négociation entre l'Espagne et la France dans la lutte contre ETA (Guittet, 2000 : 49).

19 Puisqu'elles demanderaient à être forcément très ciblées si l'organisation voulait s'en prendre aux responsables de la coopération antiterroriste. Elles ont fait quatre victimes. 
Les analyses statistiques réalisées par Sánchez-Cuenca (2009) ont montré que la violence d'État et la violence paramilitaire ont eu des effets ambivalents sur ETA. La variable "GAL " semble ne pas avoir d'effet statistique sur les actions d'ETA alors que tel n'est pas le cas des attentats menés par l'extrême droite ${ }^{20}$. Cette absence de corrélation tient au caractère restrictif de la variable "GAL " et au fait que Sánchez-Cuenca ne s'intéresse qu'aux victimes, alors que la focale portée sur les actions met en évidence des résultats opposés. L'organisation fait le choix d'attaquer les intérêts de l'État français sur le sol espagnol - dans un jeu de miroir- sans faire de victimes, dans la mesure où elle ne se place pas dans une logique d'action indiscriminée. Faute de pouvoir cibler les responsables et les exécutants de la guerre sale pour les neutraliser, ETA fait le choix de s'attaquer aux intérêts économiques de la France en forme de représailles mais sans faire de victime puisque la population n'est nullement tenue pour responsable de ces décisions politiques. En outre, ces responsables et exécutants sont assez peu nombreux. Lorsque les opérations aboutissent -ce qui est rare- elles ne produisent pas un différentiel dans le nombre de victimes causées par le groupe surtout comparé aux actions menées contre la police. On compte sept exécutions entre 1983 et 1987 à mettre au compte de la réponse à l'antiterrorisme ${ }^{21}$.

L'implication de la France contre ETA ne se résume pas à avoir fermé les yeux sur les opérations menées par les GAL sur son territoire. Entre 1984 et 1986, les autorités françaises déploient des mesures visant à déstabiliser la communauté basque espagnole installée au nord de la frontière : limitation voire non renouvellement des permis de résidence, interdiction de séjour dans les départements frontaliers, déportations dans des pays tiers (Panisello Sabaté, 2014), expulsions, extraditions en Espagne, poursuites judiciaires (Massey, 2010 : 79 et chap. 4). Les FSE, légales et illégales, opèrent sur le territoire français pendant une décennie (ibidem : 182-195) avant que ne soit formalisée leur présence légale au début des années 1990 à l'occasion notamment de l'accord antiterroriste signé le $1^{\text {er }}$ décembre 1993 entre les deux pays. Y compris après la disparition des GAL dont le dernier attentat revendiqué a lieu le 17 février 1986, les missions clandestines espagnoles anti-ETA se poursuivent en France.

Durant les années 1984-1991, l'attaque contre les intérêts français ( $N=309)$ se situe à un niveau comparable à celle contre les FSE qui essuient 353 opérations, dont 118 contre les forces armées et 235 contre la police. Les représailles contre la France sont donc portées à un niveau très élevé, celle-ci étant considérée, au même titre que l'État espagnol, comme un ennemi du mouvement indépendantiste (abertzale) et de ses militants. Les effets contreproductifs de l'antiterrorisme transpyrénéen, dans cette première phase au moins et dans ses aspects illégaux, sont ainsi manifestes. A contrario, la mise en place de la coopération antiterroriste légale, qui s'illustre notamment dans l'arrestation de Bidart, est concomitante d'une baisse de ce type d'opérations. Celle-là peut toutefois s'expliquer par une évolution des choix stratégiques de l'organisation se reportant notamment sur d'autres terrains d'action.

20 Sánchez-Cuenca (2009) ne précise pas comment sont construites les variables GAL et " extrême droite ". L'auteur souligne à juste titre que cette absence de corrélation ne signifie pas que les actions des GAL sont sans conséquence puisqu'elles ont, sur le court et le moyen terme, renforcé le soutien à ETA et ébranlé la légitimité du gouvernement espagnol au Pays basque.

21 Quand ETA tue 105 policiers sur la même période et 33 militaires. 


\subsection{ETA et les mouvements sociaux}

L'intérêt porté aux victimes du terrorisme d'ETA a pour effet d'occulter certains de ses fronts d'action, en particulier celui des luttes sociales dont certaines présentent des dimensions écologistes. L'année 1981, par exemple, présente une baisse considérable en matière de violence mortelle $(N=24)$, alors qu'on dénombre 144 actions au cours de cette même année. La plus connue d'entre elles est celle menée contre le projet de centrale nucléaire à Lemoiz, dont on a principalement retenu les cing personnes tuées à cette occasion, entre 1978 et $1982^{22}$. Le 3 août 1972, le conseil municipal de Mungia accorde à l'entreprise Iberduero " en principe et à titre provisoire l'autorisation pour le début des travaux de construction de la centrale nucléaire " sur la commune de Lemoiz ainsi qu' " une licence provisoire pour l'installation et l'ouverture de l'industrie en question ", en dépit du plan d'aménagement du territoire qui place Lemoiz en commune constituée de terrains agricoles $^{23}$. Une mobilisation populaire contre ce projet émerge à partir de juin 1974. En mai 1976, se constitue la "Commission de défense pour une côte basque non nucléaire ". Une manifestation d'ampleur a lieu sur le même thème dès le 29 août, rassemblant 50000 personnes entre Plentzia et Gorliz. Une première action illégale est menée contre les bureaux d'Iberduero à Tolosa le 18 janvier 1978 par un commando autonome. ETA entre en scène le 3 juin 1977 à Urizar de Lemoiz en plaçant un explosif dans la cantine des ouvriers de la centrale (López Romo, 2008). L'opération ne produit que des dégâts matériels ${ }^{24}$. À la suite d'une lutte de plusieurs années, un moratoire est décidé le 28 mars 1984 puis le projet est définitivement abandonné.

L'identification du nombre d'actions menées par ETA contre le maître d'œuvre du projet de centrale présente des difficultés spécifiques. À l'été 1981, Iberduero affirme avoir déjà subi 250 attentats et deux milliards de pesetas de pertes financières (EI País, 15/07/1981). S'y ajoutent près d'une centaine d'autres attentats au cours des mois suivants (Casanova, 2007 : 199) ce dont ne fait état aucune des bases de données existantes. En reconstituant partiellement ces événements, nous comptabilisons un total de 276 actions entre l'entrée d'ETA dans le

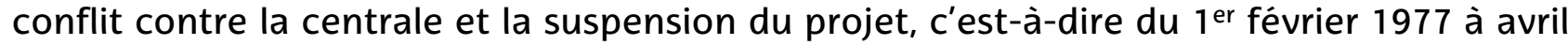
$1982^{25}$. Durant cette période, il apparaît avec évidence que l'essentiel de l'énergie déployée par ETA se concentre sur ce front (voir Tableau 3).

22 En l'occurrence, les ingénieurs José María Ryan et Ángel Pascual ainsi que les trois ouvriers Andrés Guerra, Alberto Negro et Ángel Baños.

23 Sur le sujet, voir les éléments d'informations très détaillés sur http://euskaletxeak.org/lemoiz/inicio.html

24 D'autres sources, plus attentives aux victimes, considèrent que la première opération d'ETA a lieu le 18 décembre 1977 lors de laquelle David Álvarez Peña, membre du groupe, est blessé par la garde civile puis décède des suites de ses blessures le 14 janvier 1978.

25 Notre évaluation est certainement sous-évaluée mais la campagne anti-ETA de la part des partisans, liés d'abord au régime franquiste puis au gouvernement espagnol, peut faire douter de la crédibilité des déclarations d'Iberduero dans la presse. De même les militants interrogés lors de l'enquête que nous avons menée au Pays basque entre 2017 et 2020, et qui nous a permis de rencontrer plus de 60 ex-participants de la lutte armée clandestine (Guibet Lafaye, 2020) ne s'accorde pas tous sur l'ampleur des actions menées par leur organisation. 
Tableau 3

Comparaison du nombre d'actions entre 1977 et 1983

\begin{tabular}{|l|c|}
\hline \multicolumn{1}{|c|}{ Catégories } & \multicolumn{1}{|c|}{$\begin{array}{c}\text { Nombre } \\
\text { d'actions }\end{array}$} \\
\hline Infrastructure26 (lutte sociale) & 276 \\
\hline FSE & 264 \\
\hline Banques & 124 \\
\hline Intérêts français & 40 \\
\hline Impôt révolutionnaire & 48 \\
\hline
\end{tabular}

Si l'on considère les champs où ETA est le plus intervenue au cours de ces années, il apparaît que celui de la lutte contre la centrale parvient à des niveaux supérieurs aux actions contre les FSE. On peut également faire l'hypothèse que les attaques contre les banques ont partiellement servi aux financements des opérations contre le projet de centrale. En dépit de l'intensité de ces actions, qui représente $26 \%$ de celles réalisées entre 1977 et 1983, la littérature scientifique ne propose qu'un article évoquant l'implication d'ETA dans la lutte anti-nucléaire (López Romo, 2008) ${ }^{27}$. On s'est interrogé sur les raisons du recul de la violence mortelle en 1981, lequel concerne également les autres formes de violence politique illégale (extrême droite et extrême gauche) ainsi que la répression étatique (SánchezCuenca, 2009 : 20). Le coup d'État manqué du 23-F a été désigné à tort comme la cause de la diminution de la violence meurtrière (Martínez Herrera, 2007). Le fait qu'ETA-pm tende à renoncer à ce type de moyens d'action a également été mis en avant (Sánchez-Cuenca, 2009 : 20). Pourtant, comme le reconnaît Sánchez-Cuenca, "il n'y a pas de preuve suffisante pour croire que la tentative de coup d'État a eu un effet causal direct sur l'activité d'ETA " (ibidem : 20). Celui-là estime que " la diminution des attentats meurtriers en 1981 est le résultat de l'incapacité d'ETA à se remettre de ces nombreuses arrestations ${ }^{28}$ (ibidem : 21). Le nombre d'actions réalisées dément la baisse de l'opérationnalité du groupe, y compris à une époque où les membres d'ETA-pm tendent à se placer en retrait de la lutte. Se notent bien plutôt un changement stratégique et une réorientation des énergies clandestines vers un conflit social et environnemental, celui de la centrale nucléaire de Lemoiz. L'intérêt pour les victimes, causées par ETA, occulte son implication dans une lutte politicosociale, où le choix stratégique consiste à venir en soutien de la lutte sociale. L'implication du groupe armé dans ce type de combat peut être interprétée comme opportuniste -en venant en appui d'une lutte populaire, le groupe cherche à accroître le soutien social qui lui est dévolu-, idéaliste - visant à défendre un Pays basque préservé des effets délétères de l'énergie nucléaire-, stratégique —dans son opposition à l'État espagnol qu'elle finit par faire

26 Par « infrastructure ", nous désignons conformément à la définition du Trésor de la Langue Française, l'« ensemble des installations, des équipements permanents qui conditionnent le fonctionnement d'un organisme ou d'une entreprise, l'activité économique d'une région, d'un pays. Infrastructure culturelle, routière, sanitaire ; infrastructures de communication, de transport. Infrastructure rurale (irrigation, arasement des talus ou haies, reboisement, etc.) ".

27 Tejerina (2015) fait cependant mention du sujet.

28 Le ministère de l'Intérieur fait état de 714 personnes arrêtées, contre une moyenne annuelle de 222. 
fléchir-, politique-contre une entreprise dont les proximités avec la municipalité franquiste était connues ${ }^{29}$.

Bien que l'on puisse considérer qu'il s'est agi pour ETA de se maintenir sur la scène publique et politique par ce type d'engagement, la lutte pour la préservation écologique a constitué une constante de sa stratégie. À la suite de la campagne contre les intérêts français, ETA se distingue d'abord dans l'opposition à la construction d'une autoroute traversant la vallée de Leizarán entre 1988 et 1991 puis contre le train à grande vitesse, dit Y vasca, au cours des années 2000.

L'autoroute de Leizarán devait relier le Guipúzcoa et la Navarre. Les travaux ont commencé en Navarre en août 1989 et en Guipúzcoa en mai 1991. La coordination Lurraldea s'est constituée en opposition à ce projet et a milité contre ce dernier entre 1985 et 1991. Elle est parvenue à obtenir la modification du tracé de l'autoroute, inaugurée en mai 1995. La célébration de ce succès par Herri Batasuna, le 22 avril 1992, a été largement relayée par la presse (El Correo, 4/12/2008). ETA a secondé au plan militaire les démarches entreprises par Lurraldea ${ }^{30}$. Comme dans le cas de Lemoiz, l'identification du nombre d'actions menées par ETA dans la campagne anti-autoroute est délicate. On estime qu'entre 1989 et 1991 la Coordination anti-autoroute a réalisé 158 actions de sabotage, consistant principalement en cocktails Molotov lancés contre des machines et des camions de chantier (Casanova, 2007 : 258). Ces sabotages ont commencé en 1988. On attribue à ETA plus de 200 attentats en six ans (ABC España, 9/12/2006), en lien avec ce projet d'autoroute, dont on peut se demander s'ils sont bien le fait de la seule organisation clandestine ${ }^{31}$. Nous avons, pour notre part, identifié avec assurance 114 actions menées par ETA contre le projet d'autoroute (et 46 susceptibles de s'y rapporter) entre le $11 / 09 / 1989$ et mai 1995, c'est-à-dire entre 17,6 et $24,8 \%$ du total des opérations du groupe.

\section{Tableau 4}

Comparaison des principaux pôles d'interventions d'ETA entre 1989 et 1995

\begin{tabular}{|l|c|}
\hline \multicolumn{1}{|c|}{ Principales catégories } & Total des actions \\
\hline Intérêts français & 100 \\
\hline FSE & 197 \\
\hline Actions symboliques & 26 \\
\hline Infrastructure & 46 \\
\hline Infrastructure (lutte sociale) & 114 \\
\hline Total & 646 \\
\hline
\end{tabular}

29 http://euskaletxeak.org/lemoiz/1972/1972.html

30 Ce qui a conduit au décès de deux entrepreneurs et de deux policiers.

31 La même source évoque la kale borroka, c'est-à-dire les formes de la guérilla urbaine qui ont surgi au début des années 1990 au Pays basque, et les " groupes Y ", dits groupes d'appui d'ETA. Nous avons fait le choix de dissocier l'action de la kale borroka de celle d'ETA car les liens entre les deux entités continuent de faire l'objet de discussions, en particulier d'enjeux politiques où la part de l'information validée et celle d'hypothèses à charge non vérifiables demeurent difficiles à dénouer. Il est loin d'être acquis ni scientifiquement établi que la kale borroka agisse au nom d'ETA. II n'est pas certain non plus que les " groupes $Y$ " coïncident avec une réalité constituée plutôt qu'avec une fiction antiterroriste. Sur la Kale Borroka et le mouvement indépendantiste basque, voir : Ferret (2016). 
Bien qu'au cours de ces années, l'action contre les FSE mobilise les forces vives du groupe avec $30,5 \%$ des opérations menées contre elles, l'implication dans les luttes sociales et écologistes est indéniable. II y a incontestablement un enjeu sociopolitique pour ETA à s'impliquer dans ce type de conflit qu'il soit question de revendiquer la défense du Pays basque, un souci de la préservation écologique, mais également la nécessité de constituer une opposition ferme contre l'État espagnol, associée à une volonté de mettre en évidence sa faiblesse. L'opposition au projet d'autoroute aboutit finalement à une solution de compromis consistant dans la modification de son tracé.

Les dissensions autour du projet ferroviaire de train à haute vitesse, dit " $Y$ vasca ", devant relier Madrid-Vitoria-Dax, en passant par les trois capitales basques, constitue une nouvelle occasion pour ETA d'intervenir dans la lutte contre les "Grands projets inutiles " et le combat écologiste. La décision de réaliser la "Y vasca " est validée par Madrid le 24 avril 2006. L'essentiel des actions d'ETA contre cette dernière est concentré en 2008 avec 4 opérations dont une mortelle (sur les 34 réalisées dans l'année), ce qui représente 11,8\% des actions menées en 2008. Durant cette année, les cibles sont plutôt d'ordre touristique et en lien avec les FSE, ETA renouant avec une stratégie mise en œuvre aux origines de ses opérations.

\subsection{Les "Campagnes d'été "}

L'attaque contre les structures de l'État, menée par ETA, n'a pas seulement visé les FSE ou la structure politique de l'État espagnol mais également ses intérêts économiques, avec pour intention assumée à la fois d'affaiblir ses soubassements financiers et de rappeler à l'opinion internationale le conflit au Pays basque. Pour ce faire, le groupe a procédé dès la fin des années 1970 à ce que l'on nomme les "campagnes d'été " visant les intérêts touristiques espagnols et déplaçant partiellement l'exercice de la violence politique du Pays basque vers la côte méditerranéenne. Ces campagnes interviennent de façon cyclique et récurrente dans la stratégie d'ETA contre l'État espagnol. Elles sont souvent le fait de commandos itinérants, tels le " commando des Français " actif en 1993. À la différence de l'attaque contre les intérêts français, ces campagnes n'interviennent pas durant une période spécifique, précisément délimitée dans le temps, mais de façon ponctuelle et récurrente.

Tableau 5

Proportions des actions (en \%) des « campagnes d'été " d'ETA sur l'ensemble de ses opérations

\begin{tabular}{|c|c|}
\hline Périodes & Pourcentage des « campagnes d'été » \\
\hline $1979-1980$ & 9,5 \\
\hline $1985-1986$ & 24,9 \\
\hline 1993 & 23,5 \\
\hline 1996 & 34,0 \\
\hline $2002-2005$ & 17,5 \\
\hline 2004 & 30,3 \\
\hline 2008 & 23,5 \\
\hline
\end{tabular}


La fragilisation des intérêts économiques espagnols constitue une constante de l'action du groupe au même titre que l'attaque contre les FSE. Les deux nuisent de surcroît à l'image internationale de l'Espagne. Sans qu'elle ait été comprise dans sa logique stratégique, la place de ces " campagnes d'été " a été mise en lumière par l'analyse statistique de Barros Passos et Gil-Alana (2006) qui, dans leur comparaison des périodes de cessez-le-feu et des actions armées, notent que les attaques d'ETA semblent augmenter en été. Ces opérations se comprennent comme un déplacement de l'intervention armée vers des cibles plutôt spectaculaires, de forts retentissements mais avec des moyens réduits et faisant peu de victimes. Elles consistent le plus souvent à déposer des engins explosifs sur des plages ou devant des hôtels, à attenter aux infrastructures de transport (notamment ferroviaire comme en août 1991 et 1993), à avertir les secours en amont puis à guider les FSE vers les objets. Ces interventions adviennent à partir des années 1990 dans un contexte où l'ensemble des opérations d'ETA s'est ralenti. Elles présentent un profil particulier puisque leur caractère cyclique contraste avec le fait qu'elles ne représentent que 6,3\% du total des actions d'ETA sur l'ensemble de son histoire et n'ont fait qu'une victime mortelle, ce qui explique qu'elles soient passées inaperçues des exploitations statistiques précédemment réalisées (voir Tableau 7).

\subsection{Guerre d'usure vs. contrôle de la population 32 ?}

La focale portée sur les victimes permet à Sánchez-Cuenca (2009) de situer son interprétation dans l'interrelation organisation clandestine/action gouvernementale, là où en adoptant un point de vue centré sur le groupe illégal d'autres perspectives se dessinent. La première focale conduit à interpréter la deuxième phase du déploiement de la violence d'ETA (après 1977) en termes de "guerre d'usure " (Sánchez-Cuenca, 2001 : chap. 3 ; Sánchez-Cuenca, 2007 ; Sánchez-Cuenca, 2009 : 4). Au cours de cet intervalle, ETA n'attendrait plus un soulèvement des masses mais aurait décidé d'utiliser la violence comme un moyen d'infliger des pertes à l'État qui refusait de satisfaire ses revendications, fondées sur l'alternative KAS. « Durant cette période, ETA agit conformément à l'hypothèse que si le coût infligé à l'État, en termes de vies humaines, de pertes financières et de destruction matérielle, est suffisamment élevé, l'État finira par abandonner le combat " (Sánchez-Cuenca, 2009 : 4-5), conformément à la définition de la guerre d'usure (De la Calle et Sanchez-Cuenca, 2006 : 18-21).

Pourtant, comme nos analyses l'ont suggéré, l'interprétation de l'action armée d'ETA à partir du seul binôme de la guerre d'usure et du contrôle de la population - développée par De la Calle et Sanchez-Cuenca (2006) - méconnaît les nuances de l'évolution stratégique du groupe $^{33}$. Il s'est bien agi, dans l'essentiel des phases historiques de ce collectif, d'instaurer un rapport de force avec l'État espagnol afin, dans un premier temps, de parvenir à une autonomie puis en vue d'obtenir une négociation, sachant que l'objet de cette dernière a varié avec

32 Cette double expression est inspirée des catégories analytiques mobilisées par De la Calle et Sanchez-Cuenca (2006).

33 En particulier, le fait de considérer que l'atteinte aux FSE est comparable à celle d'hommes politiques ou de représentants de l'État, en Espagne ou à l'extérieur du pays, qui permet d'étendre les limites temporelles de la guerre d'usure occulte, d'une part, l'option stratégique et politique de rupture que constitue l'atteinte à des civils. Il néglige en outre la réaction négative qu'ont suscitée les exécutions de conseillers municipaux dans la population. L'embarras de De la Calle et Sanchez-Cuenca est manifeste quant à la périodisation de la " guerre d'usure " (2004: 69). 
le temps (un statut politique pour le Pays basque, l'amnistie, le statut des prisonniers, leur libération). Toutefois la référence exclusive à ce binôme occulte les luttes sociales menées par le groupe ainsi que ses réactions aux politiques antiterroristes de l'État espagnol, quand bien même il y aurait un effet stratégique de soutien aux luttes sociales, dans une logique de rapport de force avec l'État (voir le cas de Lemoiz et de l'autoroute traversant la vallée de Leizarán).

S'il est indéniable que l'exécution de trafiquants de drogue participe du " contrôle de la population ", généraliser cette qualification pour décrire les stratégies du groupe parait inadéquat $^{34}$. En effet ETA bénéficie, dès son émergence et jusqu'à une époque avancée de son histoire, d'un très large soutien populaire au Pays basque. À la fin des années 1990 encore, une large part de la population du territoire se mobilise pour trouver une issue au conflit dans une logique de dialogue avec ETA et de solution multipartite. Les scores réalisés par Herri Batasuna (HB) en attestent également ${ }^{35}$. La population basque est en outre consciente du caractère ciblé des attentats qui ne la mettent nullement en danger. De surcroît, à partir de la toute fin des années 1970 mais surtout dans les années 1980, les attaques à la voiture piégée se déplacent vers la capitale ${ }^{36}$, des bombes sont posées en Catalogne et sur la côte sud de l'Espagne. Le champ d'impact d'actions pouvant faire des victimes involontaires se déplace vers I'Espagne plutôt que le Pays basque, fragilisant la thèse du " contrôle de la population ".

De la même façon, on ne peut assimiler sous la catégorie " attaques de contrôle de la population " des actions dont les visées sont sans commune mesure. De la Calle et Sanchez-Cuenca (2006) estiment qu'elles concernent toutes des " collaborateurs et informateurs de la police, personnes d'idéologie d'extrême droite, personnes impliquées dans une guerre sale, petits trafiquants de drogue, hommes d'affaires qui ne paient pas l'impôt révolutionnaire ou ravisseurs qui ne paient pas la rançon exigée, travailleurs ou hommes d'affaires de la centrale nucléaire de Lemoiz, politiciens du Pays Basque, civils qui s'opposent à ETA, membres ou anciens membres d'ETA, et erreurs dans lesquelles un civil est confondu avec un autre civil ", qui ont en commun d'être des civils. Pourtant, à travers ces opérations se jouent des intentions bien distinctes qu'elles soient de guerre contre l'ennemi (pour les collaborateurs de la police, l'extrême droite et les paramilitaires), de financement de l'organisation, de soutien aux mouvements sociaux (notamment contre le projet de centrale nucléaire de Lemoiz ou contre l'autoroute de la vallée de Leizarán). Enfin le binôme " guerre d'usure vs. contrôle de la population " ne permet pas de rendre compte des ajustements stratégiques qu'ont imposé les interventions militaires de l'islam politique violent en Europe occidentale en 2001 puis en 2004 en Espagne, caractérisées par des actions indiscriminées. Cette période est ignorée par les auteurs car, du fait de leur option méthodologique (consistant à s'intéresser aux victimes), elle sort de leur champ d'attention au motif qu'elle participerait de ce qu'ils nomment de façon générique le « déclin et la fin d'ETA ».

34 Les actions contre le trafic de drogue, au-delà des exécutions ( $N=49,5,8 \%$ ) représente 3,1 \% du total des opérations entreprises par ETA. L'exécution de journalistes $(N=5)$ s'inscrit dans une logique de représailles face à des acteurs qui ont fait le choix de "pactiser avec l'ennemi " (voir l' " accord pour les libertés et contre le terrorisme » du 12 décembre 2000). Étant assimilés à ce dernier, ils sont sujets au même type d'actions.

35 HB est une organisation politique basque fondée en 1978. Le projet du parti est de nature indépendantiste et socialiste. HB a souvent été considéré comme la " vitrine " politique d'ETA. Aux élections du Parlement basque, HB s'affirme entre 1980 et 1998 comme la $2^{\text {ème }}$ ou la $3^{\text {ème }}$ force politique de la région puis en 2001 comme la $4^{\text {ème }}$. Tel est également le cas des élections aux assemblées générales du Pays basque de 1979 à 1999.

36 La première explosion à la voiture piégée a lieu à Madrid le 25 avril 1985 (EI País, 11/03/2004). 
La comparaison de bases de données, fondées sur des options méthodologiques distinctes, a permis de reconsidérer les étapes de l'histoire d'ETA. S'il convient de parler de " guerre d'usure " dans le rapport avec l'État espagnol, celle-ci ne coïncide pas avec la période 19821992, comme le suggéraient De la Calle et Sanchez-Cuenca (2006), mais avec la période 19881991. Attentive aux actions menées par ETA plutôt qu'aux victimes induites, nous avons montré que les périodes d'intense activité du groupe convergeaient avec des phases d'instabilité politique au sein de l'État espagnol, mais également avec une étape d'antiterrorisme illégal, porté par le gouvernement socialiste de Felipe González, associée à la coopération antiterroriste internationale. Elles s'incarnent en second lieu dans l'implication d'ETA dans les luttes sociales à partir des années 1970 et surtout dans les réponses stratégiques que l'organisation proposent aux aléas des négociations politiques avec l'État espagnol, en vue d'apporter une réponse à la question basque. Ces considérations permettent de déplacer l'appréhension du groupe comme une organisation terroriste - sans que nous contestions ses victimes - vers celle d'un groupe politique clandestin qui subordonne l'action armée à une stratégie sociopolitique qu'il s'agisse d'ETA-pm ou d'ETA-m.

La prise en considération du positionnement politico-stratégique du groupe permet également d'ouvrir l'approche analytique au-delà du binôme " guerre d'usure vs. contrôle de la population " comme axe explicatif de l'histoire d'ETA. Sánchez-Cuenca (2009) postule, à partir de l'analyse de la base des victimes, une fin des activités d'ETA en 1992. A contrario, nous avons souligné la persistance d'une importante activité de l'organisation après 1992, associée à un glissement du terrain des opérations militaires, à partir de cette date, de l'attaque contre les intérêts français vers une lutte contre l'autoroute traversant la vallée de Leizarán. L'arrestation du collectif Artapalo en 1992 ne signe donc aucunement la fin d'ETA. Les politiques antiterroristes, limitées aux arrestations de membres présumés d'ETA, comptent assez peu dans les évolutions stratégiques du groupe contrairement à d'autres formes, plus élaborées, d'antiterrorisme telles que les politiques d'alliance antiterroriste entre le PNV et le PSOE, celles s'imposant aux médias, ou l'absence d'évacuation d'Hipercor. De même, I'histoire d'ETA ne peut être comprise indépendamment du contexte international et de sa prise en compte par le groupe. Celle-ci l'oblige à ajuster ses moyens d'action à une opinion publique moins tolérante à la violence politique ainsi qu'au développement de l'islam politique, employant cette dernière de façon indiscriminée.

\section{RÉFÉRENCES}

Barros, C. (2003). An Intervention Analysis of Terrorism: The Spanish ETA Case. Defence and Peace Economics, 14(6), 401-412.

Barros, C., Passos, J., et Gil-Alana, L. (2006). The timing of ETA terrorist attacks. Journal of Policy Modeling, 28, 335-346.

Benford, R.D., et Snow, D.A. (2012). Processus de cadrage et mouvements sociaux : présentation et bilan. Politix, 3(99), 217-255. 
Carmena, M., Landa, J.M., Múgica, R., et Uriarte, J.M. (2013). Informe-base de vulneraciones de derechos humanos en el caso vasco (1960-2013). Vitoria-Gasteiz: Secretaría General de Paz y Convivencia. https://www.irekia.euskadi.eus/uploads/attachments/3214/ informe_base_es.pdf?1371196800.

Casanova Alonso, I. (2007). ETA 1958-2008. Medio siglo de historia. Tafalla (Navarre): Txalaparta.

Davidshofer, S., Tawfik, A., et Hagmann, J. (2016). Analyse du champ de la sécurité en Suisse : vers une hypertrophie de la sécurité intérieure et autres réflexions méthodologiques . Cultures $\in$ Conflits, 2(102), 59-93.

De la Calle, L., et Sánchez-Cuenca, I. (2006). The Production of Terrorist Violence : Analyzing Target Selection within the IRA and ETA. Madrid: Working Paper, Instituto Juan March, 230.

Desrosières, A. (2008). Gouverner par les nombres : L'argument statistique II. Paris : Presses des Mines.

Ferret, J. (2016). Crisis social, movimientos y sociedad en España hoy. Zaragoza : Sibirana Ediciones.

Fonseca, C. (2014). Informe sobre la situación procesal de los atentados perpetrados por organizaciones terroristas con resultado de muerte entre 1960 y 2014. Caso vasco. Vitoria-Gasteiz: Secretaría General para la Paz y la Convivencia.

Foucart, T. (2001). L'argumentation statistique dans la politique sociale. Mathématiques et sciences humaines, 155-156(Automne-Hiver), http://journals.openedition.org.inshs.bib. cnrs.fr/msh/2853.

Foucault, M. (2004). Sécurité, territoire, population. In Cours au Collège de France 1977-1978, leçons des 8, 15 et 22 mars 1978. Paris : Seuil.

François, A., et Machiels, Ch. (2007). Une guerre de chiffres. Histoire $\in$ mesure, XXII(2), 103-134.

Giacopucci, G. (1997). ETA. Historia política de una lucha armada, Tafalla : Txalaparta.

Goffman, E. (1974). Frame Analysis: An Essay on the Organization of the Experience. New York: Harper Colophon.

Gouvernement basque (2016). Clarificación del pasado. In Compilación de documentos generados por el Gobierno Vasco en la Legislatura 2012-2016 en materia de paz y convivencia. Vitoria-Gasteiz: Secretaria General para la Paz y la Convivencia.

Guibet Lafaye, C. (2020). Conflit au pays basque : regards des militants illégaux. Bruxelles: Peter Lang.

Guibet Lafaye, C., et P. Brochard (2020). Conséquences de choix méthodologiques sur l'analyse de l'évolution de l'organisation clandestine ETA. Studia Europa, 2.

Guibet Lafaye, C., et P. Brochard (2021). Methodological approaches to the evolution of a terrorist organisation: ETA, 1959-2018. Quality $\in$ Quantity [À paraître].

Guittet, E. (2000). Raison et déraison d'État : les GAL (Grupos Antiterroristas de Liberación), 19831987. Mémoire de DEA inédit, Université Paris X-Nanterre, Paris.

Lopez Garrido, D. (1987). El aparato policial en España. Barcelona: Ariel. 
López Romo, R. (2008). Tiñendo la patria de verde y violeta. La relación del nacionalismo vasco radical con los movimientos antinuclear y feminista en la transición. In E. Nicolás et C. González (Eds.), Ayeres en discusión. Temas clave en Historia Contemporánea hoy. Murcia: Universidad de Murcia.

Martínez Herrera, E. (2007). Government restructuring and reallocation of resources in the face of ethno-nationalist insurgency in the Basque Country (1979-2005). In M. Oberg et K. Strom (Dirs.), Resources, Governance Structures and Civil Conflict (pp. 101-124). Londres: Routledge.

Massey, J. (2010). ETA histoire secrète d'une guerre de cent ans. Paris: Flammarion.

McCaffery, P., Richardson, L., et Bélanger, J. (2016). Classification and Collection of Terrorism Incident Data in Canada. Perspectives on Terrorism, 10(5), 42-50.

Miller, E. (2016). Patterns of Collective Desistance from Terrorism: Fundamental Measurement Challenges. Perspectives on Terrorism, 10(5), 5-21.

Nuñez Astrain, L.C. (Dir.) (1993). Euskadi Eta Askatasuna, tomes I-VIII. Tafalla: Txalaparta.

Panisello Sabaté, S. (2014). La internacionalización de la política antiterrorista. VI Congreso de la Asociación de Historiadores del Presente. La apertura internacional de España: entre el franquismo y la democracia, 1953-1986, Valladolid et Madrid.

Samson, D. (2014). Usages de la statistique dans les controverses socio-techniques : une technique juridico-politique d'objectivation ? Cahiers Droit, Sciences $\in$ Technologies, 4, 87106.

Sánchez-Cuenca, I. (2001). ETA contra el Estado: las estrategias del terrorismo. Barcelona: Tusquets.

Sánchez-Cuenca, I. (2007). The Dynamics of Nationalist Terrorism: ETA and the IRA. Terrorism and Political Violence, 19(3), 289-306.

Sánchez-Cuenca, I. (2009). Explaining temporal variation in the lethality of ETA. Revista Internacional de Sociología, 67(3), 609-629.

Sánchez-Cuenca, I. (2010). The persistence of nationalist terrorism: the case of ETA. In K. Mulaj (Ed.), Violent Non-State Actors in Contemporary World Politics (pp. 69-92). New York: Columbia University Press.

Sánchez-Cuenca, I., et Aguilar, P. (2009). Terrorist Violence and Popular Mobilization. The Case of the Spanish Transition to Democracy. Politics E Society, 37(3), 428-453.

Speich Chassé, D. (2017). Les statistiques comme mode de communication politique. Le cas des premiers plans de développement au Kenya. Politique africaine, 1(145), 85-108.

Tejerina, B. (2015). Nacionalismo, violencia y movilización social en el País Vasco. Factores y mecanismos del auge y declive de ETA. Papeles del CEIC, 3(136).

Tilly, Ch. (2000). Terror, Terrorism, Terrorists. Sociological Theory, 22(1), 5-13.

Truc, G. (2012). Aux victimes du terrorisme, l'Europe reconnaissante ? Portée et limites de la Journée européenne en mémoire des victimes du terrorisme. Politique européenne, 2(37), 132-154. 


\section{ANNEXES}

\section{Annexe 1. Références des sources ayant été utilisées dans la constitution de la base de données}

1. Global Terrorism Database (GTD) : https://www.start.umd.edu/gtd/

2. Sources répertoriant les victimes du conflit au Pays basque :

- La base de données réalisées par De la Calle et Sanchez-Cuenca : https://ic3jm.es/ investigacion/proyectos/explaining-terrorist-and-insurgent-behavior/, consulté le 21/12/2020.

- La liste de victimes d'ETA publiée par Calleja (1997).

- Les associations de victimes : I'Association des Victimes du Terrorisme (www.avt.org)

- Les victimes des forces de sécurité :

https://mapadelterror.com/en/ https://mapadelolvido.blogspot.com/ https://ertzaione-1.blogspot.com/ https://web.archive.org/web/20090202121213/http://www.guardiacivil.org:80/terrorismo/acciones/estadistica07.jsp http://especiales.elperiodico.com/graficosEEPI/VICTIMAS/tablavictimas.html\#ARRIBA

- Les victimes du côté abertzale ou basque : http://www.euskalmemoria.eus/

- Autres base de données recensant les victimes du terrorisme :

http://www.angelfire.com/pq/terroristas/ http://blogs.libertaddigital.com/in-memoriam/ https://victimas-de-eta.blogspot.com https://www.abc.es/especiales/eta/victimas/11.asp https://eu.m.wikipedia.org/wiki/Zerrenda:ETAk_hildakoak_(1968-1979) https://ns-markoss88.blogspot.com/

3. Quotidiens nationaux :

- https://www.elmundo.es/; https://www.elmundo.es/eta/historia/

- https://elpais.com/

- https://www.lavangarde.com/

- http://hemeroteca.lavanguardia.com/

- https://www.elcorreo.com/

- https://www.abc.es/

- https://www.laverdad.es/

- https://www.20minutos.es/

- https://www.lainformacion.com/ 
Quotidiens régionaux:

- https://www.prensaescrita.com/euskadi.php

- https://www.noticiasdegipuzkoa.eus/

- https://www.eitb.eus/es/

- https://www.elnortedecastilla.es/

- https://www.elcorreogallego.es

- https://www.diariodesevilla.es/

— https://borrokagaraia.wordpress.com/

Quotidiens français rapportant les actions du groupe :

- https://www.liberation.fr/

- https://www.lexpress.fr/

— https://www.lejdd.fr/

Sources anglophones :

— https://www.theguardian.com

— http://news.bbc.co.uk

- https://www.reuters.com

— https://www.telegraph.co.uk

Les journaux en langue basque :

—https://www.naiz.eus/

— http://gara.naiz.eus

- http://www.diariovasco.com/

4. Les sources gouvernementales du ministère de l'Intérieur espagnol :

- http://www.mir.es/DGRIS

— http://www.interior.gob.es/fallecidos-por-terrorismo

5. Les documents produits par la gauche abertzale et l'organisation clandestine elle-même

— http://www.abertzalekomunista.net/es/relato-historico/historia-del-mlnv/v-asamblea/ documentos-v-asamblea

— https://borrokagaraia.wordpress.com/acabas-de-Ilegar-empieza-aqui/

- http://www.ehk.eus/es/v-asamblea-cast/revistas-v-asamblea-cast/4292-barneko-gora-beherez-1-1968-cast

- http://euskaletxeak.org/lemoiz/inicio.html 


\section{Annexe 2}

Tableau 6

Répartition des opérations selon les périodes distinguées et en fonction des catégories de cibles

\begin{tabular}{|c|c|c|c|c|c|c|c|c|}
\hline Catégories & $\begin{array}{c}1959- \\
1974\end{array}$ & $\begin{array}{l}1974- \\
1976\end{array}$ & $\begin{array}{l}1977- \\
1980\end{array}$ & $\begin{array}{c}1981- \\
1991\end{array}$ & $\begin{array}{c}1992- \\
1997\end{array}$ & $\begin{array}{c}1998- \\
1999\end{array}$ & $\begin{array}{l}2000- \\
2001\end{array}$ & $\begin{array}{r}2002- \\
2009\end{array}$ \\
\hline Actions symboliques & 28 & 0 & 17 & 16 & 13 & 0 & 3 & 24 \\
\hline Banque & 19 & 4 & 8 & 160 & 1 & 0 & 4 & 5 \\
\hline Bâtiments publics & 58 & 2 & 61 & 30 & 9 & 0 & 12 & 26 \\
\hline Civils & 0 & 0 & 1 & 4 & 4 & 0 & 0 & 0 \\
\hline Drogue & 0 & 0 & 4 & 79 & 12 & 0 & 1 & 0 \\
\hline Entreprises (lutte sociale) & 3 & 1 & 9 & 2 & 7 & 7 & 0 & 1 \\
\hline Entreprises privées & 2 & 0 & 1 & 6 & 1 & 0 & 3 & 16 \\
\hline FSE & 15 & 20 & 169 & 448 & 83 & 2 & 28 & 25 \\
\hline Impôt révolutionnaire & 0 & 0 & 27 & 61 & 24 & 0 & 13 & 31 \\
\hline Informateurs de la police & 6 & 5 & 19 & 17 & 1 & 0 & 0 & 0 \\
\hline Infrastructure & 6 & 4 & 39 & 57 & 22 & 0 & 3 & 29 \\
\hline Infrastructure (lutte sociale) & 0 & 0 & 112 & 231 & 53 & 0 & 0 & 4 \\
\hline Intérêts américains & 0 & 0 & 0 & 6 & 0 & 0 & 0 & 0 \\
\hline Intérêts français & 4 & 0 & 27 & 322 & 10 & 0 & 1 & 0 \\
\hline Journalistes \& Media & 6 & 0 & 7 & 2 & 1 & 0 & 14 & 8 \\
\hline Lieux publics & 0 & 0 & 0 & 6 & 2 & 0 & 5 & 8 \\
\hline Personnel politique & 0 & 2 & 20 & 4 & 8 & 8 & 20 & 9 \\
\hline Prison & 0 & 0 & 0 & 10 & 5 & 0 & 2 & 0 \\
\hline Propriétés privées & 0 & 0 & 1 & 1 & 0 & 0 & 0 & 0 \\
\hline Réponse à l'antiterrorisme & 8 & 1 & 17 & 32 & 0 & 0 & 0 & 1 \\
\hline Représentants de l'État & 1 & 1 & 6 & 24 & 7 & 0 & 12 & 3 \\
\hline Réquisitions & 39 & 3 & 6 & 1 & 0 & 1 & 1 & 1 \\
\hline Tourisme & 0 & 0 & 31 & 87 & 41 & 0 & 9 & 38 \\
\hline Non identifiables & 0 & 0 & 3 & 4 & 0 & 0 & 2 & 0 \\
\hline Total & 191 & 47 & 585 & 1.610 & 304 & 18 & 133 & 229 \\
\hline
\end{tabular}




\section{Tableau 7}

Répartition en pourcentage du type d'actions sur l'ensemble de l'histoire d'ETA

\begin{tabular}{|l|c|}
\hline \multicolumn{1}{|c|}{ Catégories } & $\begin{array}{c}\text { Total des opérations menées } \\
(\%)\end{array}$ \\
\hline FSE & 25,3 \\
\hline Luttes sociales contre les infrastructures & 12,9 \\
\hline Intérêts français & 11,6 \\
\hline "Campagnes d'été " & 6,3 \\
\hline Actions symboliques & 3,2 \\
\hline Bâtiments publics & 6,4 \\
\hline
\end{tabular}

